\title{
Linking Whole-Slide Microscope Images with DICOM by Using JPEG2000 Interactive Protocol
}

\author{
Vilppu J. Tuominen ${ }^{1}$ and Jorma Isola ${ }^{1}$
}

\begin{abstract}
The use of digitized histopathologic specimens (also known as whole-slide images (WSIs)) in clinical medicine requires compatibility with the Digital Imaging and Communications in Medicine (DICOM) standard. Unfortunately, WSIs usually exceed DICOM image object size limit, making it impossible to store and exchange them in a straightforward way. Moreover, transmitting the entire DICOM image for viewing is ineffective for WSIs. With the JPEG2000 Interactive Protocol (JPIP), WSIs can be linked with DICOM by transmitting image data over an auxiliary connection, apart from patient data. In this study, we explored the feasibility of using JPIP to link JPEG2000 WSIs with a DICOM-based Picture Archiving and Communications System (PACS). We first modified an open-source DICOM library by adding support for JPIP as described in the existing DICOM Supplement 106. Second, the modified library was used as a basis for a software package (JVSdicom), which provides a proof-of-concept for a DICOM client-server system that can transmit patient data, conventional DICOM imagery (e.g., radiological), and JPIP-linked JPEG2000 WSIs. The software package consists of a compression application (JVSdicom Compressor) for producing DICOMcompatible JPEG2000 WSIs, a DICOM PACS server application (JVSdicom Server), and a DICOM PACS client application (JVSdicom Workstation). JVSdicom is available for free from our Web site (http://jvsmicro scope.uta.fi/), which also features a public JVSdicom Server, containing example $\mathrm{X}$-ray images and histopathology WSIs of breast cancer cases. The software developed indicates that JPEG2000 and JPIP provide a well-working solution for linking WSIs with DICOM, requiring only minor modifications to current DICOM standard specification.
\end{abstract}

KEY WORDS: Digital pathology, telepathology, DICOM, JPEG2000, JPIP, virtual slide, whole-slide imaging, WSI

\section{BACKGROUND}

$\mathrm{H}$ ospital information systems (HIS) have evolved from closed and proprietary-linked systems into open and standards-based. This change has encouraged medical equipment vendors and software developers to create uniform and interoperable systems. Standardized interfaces let developers link different internal department information systems together: for example, connecting the Laboratory Information System (LIS) of a clinical chemistry or a pathology department with the Radiology Information System in a radiology department. Linked data repositories make all patient-related material (e.g., clinical history and images from different modalities) available to all institution personnel. For instance, pathologists and radiologists can view breast ultrasound and X-ray images simultaneously with corresponding histological specimens.

The most widely used medical imaging standard is the Digital Imaging and Communications in Medicine $(\mathrm{DICOM})^{1}$, which is routinely used in several medical specialties, especially radiology. In pathology, digitization of whole microscope specimens has only recently become possible with high-throughput slide scanners ${ }^{2}$. The digitized versions of microscope glass slides are called "virtual slides" or "whole-slide images" (WSIs). Acquiring, handling, and displaying WSIs is commonly called "virtual

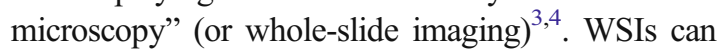
be used for local viewing or, more practically, for

\footnotetext{
${ }^{1}$ From the Institute of Medical Technology, University of Tampere and Tampere University Hospital, 33014, Tampere, Finland.

Correspondence to: Jorma Isola, Institute of Medical Technology, University of Tampere and Tampere University Hospital, 33014, Tampere, Finland; tel: +358-3-35516729; fax:+358-3-35518923; e-mail: jorma.isola@uta.fi

Copyright (C) 2009 by Society for Imaging Informatics in Medicine

Online publication 5 May 2009

doi: 10.1007/s10278-009-9200-1
} 
remote viewing by transmitting them over networks ${ }^{5}$. Within the internal network of a hospital or pathology department, personnel can use WSIs in case meetings, slide seminars, and instructional live-audience presentations ${ }^{6}$. By allowing access over the Internet, WSIs can also be used more widely in second-opinion consultations, national and international conferences, and inter-laboratory quality assurance programs ${ }^{7}$.

Since microscope specimens are often up to $20 \times$ $30 \mathrm{~mm}$ in size, a WSI can contain up to $40 \mathrm{~GB}$ of uncompressed image data (with a scanning resolution $0.2-0.5 \mu \mathrm{m}$ per pixel $)^{2}$. The amount of data increases further if scanning is done at a higher optical magnification and/or if several focus layers (along $Z$-axis) are scanned (e.g., in cytopathology $)^{8}$. Due to the large size of WSIs, all viewing systems described to date apply the "ondemand" principle: that is, only a user-requested area (with a desired resolution) of the WSI is decoded and displayed. Moreover, the large image size necessitates the use of lossy image compression. Lossy compression can yield a 10 - to 30 -fold compression ratio compared to lossless compression, without affecting the diagnostic properties of a $\mathrm{WSI}^{9}$. Thus, a suitable image format for virtual microscopy needs to be based on an effective image compression algorithm, as well as to provide a sophisticated random access technique.

Virtual microscopy currently lacks a universally accepted WSI format. There are several proprietary image formats that are tied to specific scanner vendors, such as SVS (by Aperio Technologies, USA), NDP (by Hamamatsu Photonics, Japan), and Mirax (by Carl Zeiss MicroImaging, USA). The interoperability between different vendor formats and viewing software is practically non-existent. We have previously shown that the open JPEG2000 standard is a suitable format for WSIs, allowing fast random slide access and efficient lossy compres$\operatorname{sion}^{10}$. Although JPEG2000 compression is computationally intensive, the process can be matched with current slide scanner speeds by utilizing multi-core processor environments ${ }^{10}$. JPEG2000 is a family of standards supervised by the Joint Photographic Experts Group standardization committee ${ }^{11,12}$. The standard family currently consists of 13 parts, three of which are essential for virtual microscopy. Part 1 (Core Coding System) ${ }^{13}$ specifies the codestream syntax and the JP2 file format, which uses "jp2" as the common file extension. Part 2 (Exten- sions $)^{14}$ provides extensions for the first part. Part 9 (Interactivity Tools, APIs, and Protocols) $^{15}$ introduces the JPEG2000 Interactive Protocol (JPIP) for remote serving and viewing of JPEG2000 images. We have previously developed and released a free JPEG2000 software package (called JVS, for JPEG2000 Virtual Slide) comprising WSI compression, viewing, and network server applications ${ }^{10}$. Della Mea et al. ${ }^{16}$ have presented a survey of currently available JPEG2000 viewing software.

For clinical diagnostic use, WSIs must be compatible with existing imaging standards, such as DICOM $^{17}$. Although a DICOM Object definition for visible light microscopy exists ${ }^{18}$, WSIs are too large (both pixel dimensions and byte size) to be used directly as these objects. A base standard Correction Item (CP 896) ${ }^{19}$ to overcome the image dimension limitation was recently rejected by the DICOM Standards Committee ${ }^{20}$. Nevertheless, the committee will continue to consider the possibility of introducing this change to the standard as a supplement. In addition to image size, the access characteristics of WSIs differ from conventional DICOM images. Panning and zooming within a huge WSI require fast random access with on-demand decoding. Currently, the DICOM standard includes the basic parts of the JPEG2000 standard in Supplements $61^{21}$ and $105^{22}$. Supplement 106 (JPEG 2000 Interactive Protocol) ${ }^{23}$ describes two JPIP-based Transfer Syntaxes as methods of delivering image pixel data apart from patient data: the noncompressed JPIP Referenced Transfer Syntax and the Deflate-compressed ${ }^{24}$ JPIP Referenced Deflate Transfer Syntax. Thus, the DICOM standard specification already contains necessary elements for transmitting WSIs over JPIP.

When using the JPIP Transfer Syntaxes in a DICOM-based Picture Archiving and Communication System (PACS), a DICOM server sends its client a Uniform Resource Locator (URL) string that refers to the WSI pixel data provider (i.e., a JPIP server), together with the image name, which can be arbitrary and unrelated to patient data (as shown in Fig. 1). Upon receiving the pixel data provider reference, the client DICOM workstation can either use a built-in JPIP viewer or invoke an external one for retrieving the WSI from the specified JPIP server. All network messaging between the PACS and the client end is done according to the DICOM protocol, except the JPIP transmission, which is by default performed on top of the Hypertext Transfer Protocol (HTTP/1.1 $)^{25}$ for compatibility with existing Web 


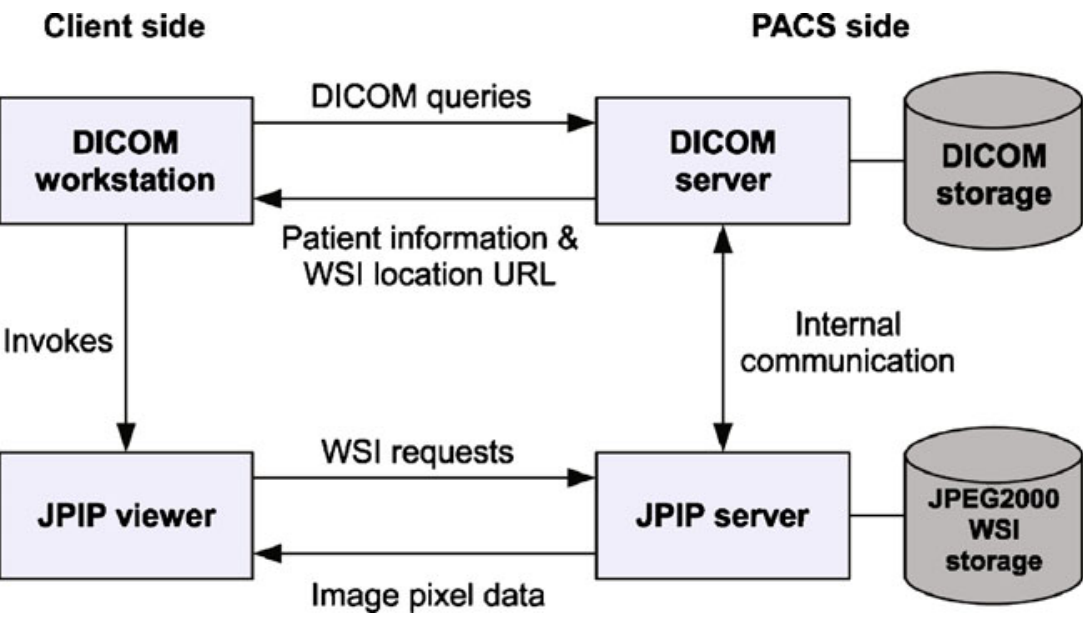

Fig 1. The principle of transmitting whole-slide images (WSIs) within a DICOM-based PACS by using JPEG2000 Interactive Protocol (JPIP).

infrastructure, but it can also be done using a lowerlevel transport protocol (such as Transmission Control Protocol, TCP) $)^{26}$. Image serving performance of JPIP has been demonstrated to be excellent and upwards scalable in multi-client systems ${ }^{10,27}$.

Although JPIP is described in the DICOM standard specification and a brief description of a commercial application exists ${ }^{28}$, we are not aware of any open DICOM software solutions or libraries supporting it. In this study, we explore the feasibility of linking JPEG2000 WSIs with a DICOM-based PACS by using JPIP. First, we modified an opensource DICOM library by adding support for JPIP as described in the Supplement 106. Second, the modified library was used as a basis for a software package (JVSdicom), which provides a proof-ofconcept for a DICOM client-server system that can transmit patient data, conventional DICOM imagery, and JPEG2000 WSIs over JPIP. The software package consists of a compression application (JVSdicom Compressor) for producing DICOMcompatible JPEG2000 WSIs, a DICOM PACS server application (JVSdicom Server), and a DICOM PACS client application (JVSdicom Workstation). Finally, we present a DICOM-compatible whole-slide imaging system based on the software developed.

\section{MATERIALS AND METHODS}

An open-source DICOM library (OFFIS DCMTK DICOM toolkit, version 3.5.4 $)^{29}$ was modified by adding support for JPIP, as described in the DICOM
Supplement 106. The modified library was used to embed DICOM functionality to the developed JVSdicom software package. JVSdicom Compressor is based on our previously described JPEG2000 compression application (JVScomp, version 2.1) ${ }^{10}$. JVSdicom Server and Workstation utilize the Qt open-source software framework (version 4.3$)^{30}$ for core application functionality and the Tango Icon Library $^{31}$ for graphical user interface elements. The software package was written in $\mathrm{C}++$ programming language and built for 32-bit Windows ${ }^{\circledR}$ platforms but can be run under a 64-bit Windows ${ }^{\circledR}$ platform as well.

Reference material representing typical diagnostic imagery of breast cancer was obtained from Tampere University Hospital. The material comprises radiology imagery (ultrasound, mammography, bone scan, and magnetic resonance imaging) and histological specimen slides (routine $\mathrm{H} \& \mathrm{E}$ stains and immunohistochemistry). The standardsized slides $(75 \times 25 \mathrm{~mm})$ were scanned with Aperio ScanScope ${ }^{\circledR}$ XT (Aperio Technologies, USA) using uncompressed BigTIFF $^{32}$ as the primary output format. Whole-mount section slides $(75 \times 50 \mathrm{~mm})$ were acquired with a Zeiss Axioskop40 microscope (Carl Zeiss MicroImaging, USA) as described equipped with a chargecoupled device color camera (QICAM Fast; QImaging, Canada) and a motorized specimen stage (Märzhäuser Wetzlar GmbH, Germany). The automated image acquisition was controlled by the Surveyor imaging system (Objective Imaging, UK) using uncompressed bitmap as the 
primary output format. The developed JVSdicom Compressor application was used to convert the histological reference material into the DICOMcompatible JPEG2000 WSI format.

\section{RESULTS}

The JVSdicom software package was developed as a proof-of-concept for a DICOM client-server system that can transmit patient data, conventional DICOM imagery (e.g., radiological), and JPEG2000 WSIs using the JPIP Referenced Transfer Syntax. The software package consists of a compression application (JVSdicom Compressor), a DICOM PACS server application (JVSdicom Server), and a DICOM PACS client application (JVSdicom Workstation).

JVSdicom Compressor is a free, command linebased image compression application capable of converting multiple image formats (e.g., BMP, PPM, and BigTIFF) into the DICOM-compatible JPEG2000 WSI format. The output JPEG2000 file follows the optimized code-stream parameterization we have previously described ${ }^{10}$. In addition to the JPEG2000 WSI file, the application generates a supplementary DICOM file containing medical information, image properties, and a JPIP server reference to the WSI file location, which is used by the DICOM client to access the WSI. The resulting DICOM file uses the General Microscopy modality ${ }^{18}$ and Visible Light Microscopic Image Information Object Definition (IOD) ${ }^{18}$ with a minimal set of required attributes.

JVSdicom Server is an open-source DICOM PACS server application that acts as a Storage Service Class Provider (SCP) and as a Query/ Retrieve SCP (Table 1). The server is capable of accepting multiple associations simultaneously.

Table 1. Main features of JVSdicom Server

List of features

Fully DICOM-compliant PACS server

Support for several Storage SOP Classes

Support for JPEG2000 WSIs with JPIP Referenced Transfer Syntax

A public mode with open access to server

Can be used as a server for JVSdicom Workstation

Open-source
Server can be configured to contain several filesystem-based storage areas with different Application Entity (AE) Titles, as well as to limit access to these areas from a predefined AE network (Fig. 2). Alternatively, the server features a public mode, which can be used to grant open access to the server. For open access, the calling AE is assumed to have a receiving Storage SCP set up. New DICOM entries can be added into the server storage with pixel data either coming from existing image files or replaced with a JPIP reference (i.e., in case of JPEG2000 WSIs). JVSdicom Server supports several Storage Service Object Pair (SOP) Classes. A comprehensive conformance statement appears in the software documentation.

JVSdicom Workstation is an open-source DICOM PACS client application that acts as a Query/Retrieve Service Class User (SCU) and a Storage SCU (Table 2). With it, users can query and retrieve images from a DICOM-compatible PACS server (Fig. 3). Users can view the images with several image enhancement options, as well as view a summary of patient- and treatment-related information. JVSdicom Workstation interacts with a DICOM server as a conventional DICOM client, but upon receiving a JPIP reference to a JPEG2000 WSI, it invokes an external JPEG2000 viewing application. The external viewing application displays the image pixel data, while JVSdicom displays the associated DICOM medical information. Thus, by having an external viewer for WSIs, users can view conventional DICOM imagery and corresponding histopathologic specimens side by side (Fig. 4). The external viewing application can be chosen by the user. The default viewer is JVSview ${ }^{10}$. Regions of interests from the DICOM images and WSIs can both be opened in a public domain image analysis software, Image $\mathrm{J}^{33}$, which features a multitude of analysis tools for medical imaging. JVSdicom Workstation is compatible with commercial-grade DICOM servers, supporting several Storage SOP Classes (a comprehensive conformance statement appears in the software documentation).

The JVSdicom software package is fully DICOMcompliant and designed to run on Windows ${ }^{\circledR} \mathrm{XP}$ but is also Windows Vista ${ }^{\mathrm{TM}}$ compatible. The binaries and source code, as well as a comprehensive conformance statement, are available on our Web site (http:// jvsmicroscope.uta.fi/). The Web site also features a public JVSdicom server, containing example images produced in the diagnostics of breast cancer. 


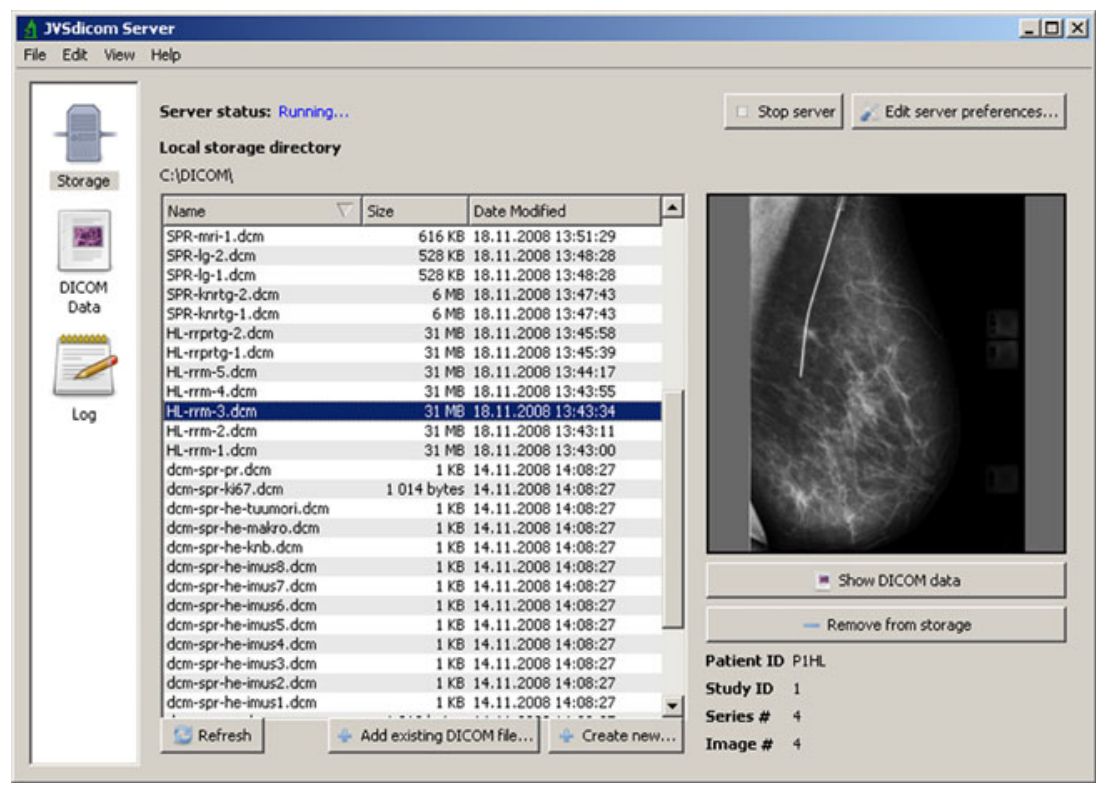

Fig 2. A screenshot of JVSdicom Server storage management view. DICOM image objects can be added to the storage by importing existing image files or by creating a new JPIP-referenced object.

\section{DISCUSSION}

We developed the JVSdicom software package to study the feasibility of linking JPEG2000 WSIs with a DICOM-based PACS by using JPIP. JVSdicom Compressor is used for creating DICOM-compatible JPEG2000 WSIs, which consist of the actual JPEG2000 image file and a supplementary DICOM file containing patientrelated information. The supplementary DICOM file can be served using the JVSdicom Server, while the JPEG2000 image files are served using a separate JPIP server. Querying and retrieving images from the JVSdicom Server can be done using the JVSdicom Workstation, which handles conventional DICOM imagery directly but uses an external JPIP client application for JPEG2000 WSI viewing.

Table 2. Main features of JVSdicom Workstation

List of features

Fully DICOM-compliant PACS client

Support for JPEG2000 WSIs with JPIP Referenced Transfer Syntax Viewing of radiological images and corresponding histological WSIs side by side

A functional link with public domain image analysis software (ImageJ)

Can be used as a client for JVSdicom Server

Open-source
A complete, DICOM-compatible whole-slide imaging system can be constructed by combining JVSdicom with our previously described JPEG2000 viewing application (JVSview) and JPIP network serving application (JVSserv; Fig. 5). In this model system, a WSI scanner produces raw image data, which is processed by JVSdicom Compressor. JVSdicom Compressor produces a JPEG2000 file containing the actual WSI image data and a DICOM file containing the associated medical data (i.e., patient information) as well as some mandatory image properties, such as width and height. By default, the produced DICOM file contains anonymized DICOM entries, but it could be linked with a LIS or a HIS for retrieving patient information. A straightforward way to name the JPEG2000 WSI file is to use the microscope slide label identification string, which can be read automatically if bar-coded labels are used. The JPEG2000 WSI file is then moved into a JVSserv server, and the DICOM file is moved into a JVSdicom Server, which both are parts of the same PACS. Both files can be stored separately inside a server-specific storage area within the PACS. JVSdicom can also receive imagery from other imaging modalities, which are in turn linked with the LIS or HIS. End-users (e.g., pathologists or physicians) query the JVSdicom Server with a JVSdicom Workstation and retrieve patient-linked 


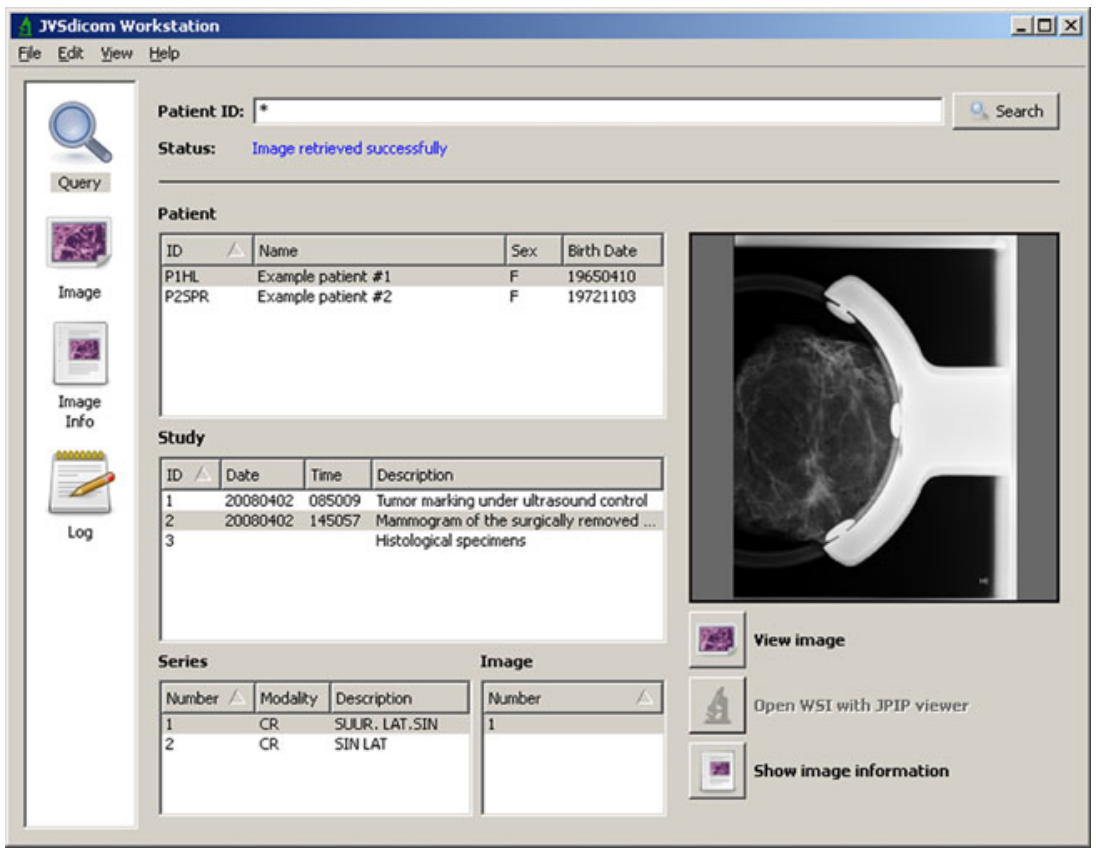

Fig 3. A screenshot of JVSdicom Workstation showing the PACS query view. Conventional DICOM imagery is displayed in the thumbnail window, while JPEG2000 WSIs are opened in an external JPIP viewer.

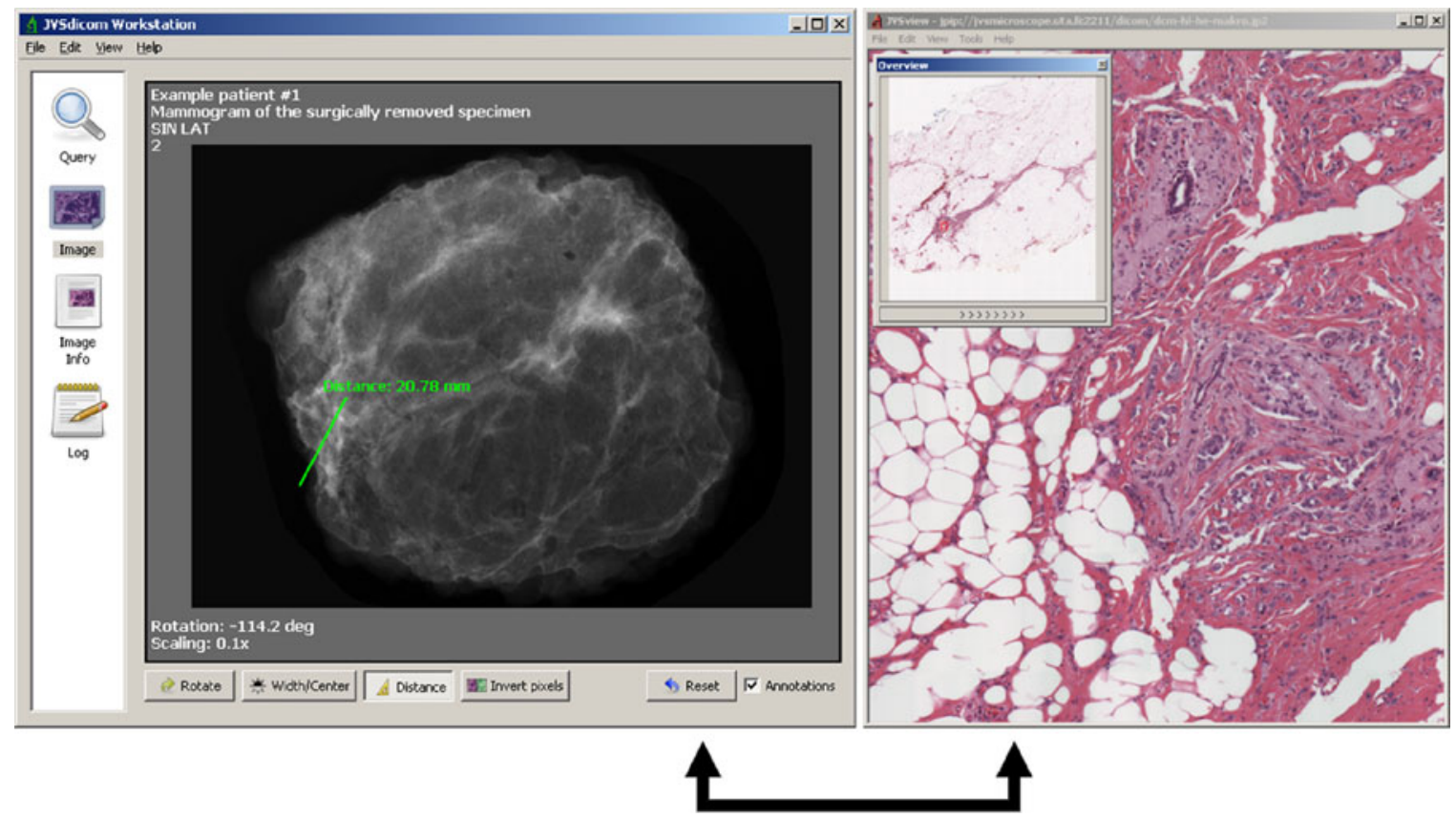

Fig 4. Viewing of a breast cancer specimen X-ray and corresponding histological WSI (whole-mount section) side by side with JVSdicom Workstation and an external JPIP viewer. Within JVSdicom, users can rotate the image, adjust width and center values, and measure distances by using the ruler tool. The JPIP viewer displays the WSI using an overview and a main navigation window. 


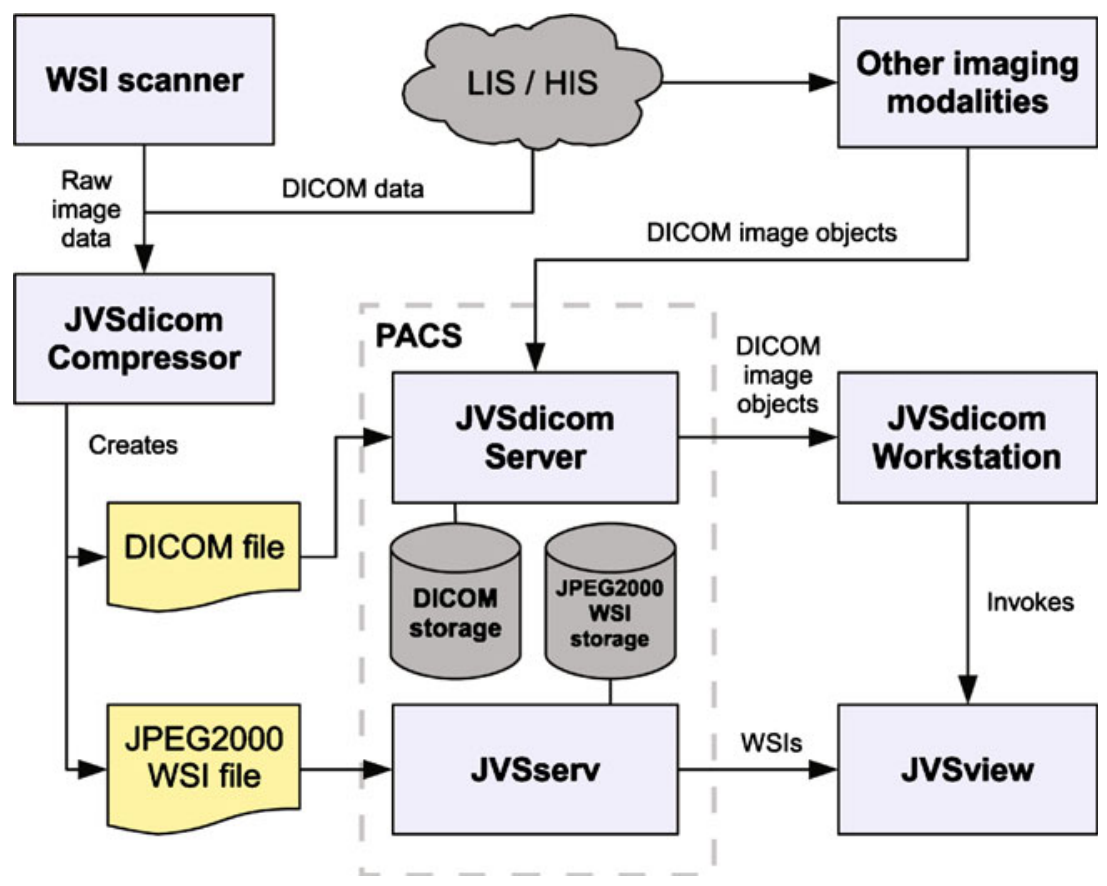

Fig 5. A model system for linking whole-slide images (WSIs) with DICOM by using JPEG2000, JPIP, and the JVS software. The WSI scanner produces raw image data, which JVSdicom Compressor processes. The resulting DICOM and JPEG2000 WSI files are moved into the PACS to JVSdicom Server and JVSserv, from which they are queried with JVSdicom Workstation and viewed with JVSview.

image objects. They can view and analyze conventional DICOM imagery within JVSdicom Workstation, while WSIs are opened with JVSview in another viewing window. The DICOM data is transmitted using the JPIP Referenced Transfer Syntax, and the JPEG2000 WSI data is transmitted via an auxiliary channel over JPIP. The system architecture makes it possible to use JVSserv separately outside the PACS, since WSIs do not contain any DICOM references.

An open issue with using JPEG2000 WSIs and JPIP is the image dimension restriction in the DICOM standard specification. Since image width and height tags are currently described using 16-bit values, oversized (width or height $>65,535$ pixels) WSIs will show false information with regards to these tags. However, since the definitive image width and height information are always also stored in the JPEG2000 code-stream header, the DICOM counterpart values can be bypassed. This requires the JPIP client software to use the code-stream values instead of the DICOM-specific values. The software described in this study follows this principle. In the future, if a DICOM Supplement equivalent to the CP 896 (to eliminate 16-bit image dimension restrictions) is introduced to the standard, the JPIP viewing software can also operate using the DICOM-specific image dimension tags. Current limitations for DICOM image object size due to 32-bit addressing (4 GB) do not affect JPEG2000 WSIs as long as they are stored in a separate JPIP server, such as the one described in this study. As such, the JPEG2000 standard does not limit individual file size and image dimensions, even in the largest future WSIs ${ }^{11,13}$.

The DICOM Working Group for pathology imaging (WG-26) is currently making an effort to consolidate pathology concepts and whole-slide imaging with $\mathrm{DICOM}^{34}$. WG-26 has recently finalized Supplement 122 (Specimen Module and Revised Pathology SOP Classes), introducing a new mechanism for pathology specimen identification in $\mathrm{DICOM}^{35}$. The new mechanism revises the concept of "specimen" within DICOM framework. Zwönitzer et al. $^{36}$ have also proposed a similar approach. The scope of our study was to exemplify a DICOM WSI solution based on JPIP. Thus, the software described does not currently implement Supplement 122.

WG-26 has also recently started preparing a draft for a new DICOM Supplement for whole-slide imaging containing an IOD and SOP Classes for WSIs (as discussed in the WG-26 meetings) ${ }^{37}$. At 
its current state, the supplement utilizes a "pyramidal" approach in which the original resolution WSI is split into small image tiles (usually thousands of them). In addition, a number of successively lower resolution versions of the original tiles may be precomputed, creating a pyramid-shaped resolution structure. All the image tiles are stored as a conventional DICOM Series and accessed with the DICOM WSI object, which provides a mapping between the Series and the conceptual image pyramid. The pyramidal approach is similar to what various WSI scanner vendors have used in their web viewing solutions for several years. Prior to its finalization, the supplement draft is subject to changes.

Both approaches for whole-slide imaging in DICOM, the pyramidal and the JPIP-based, as described in this study, can be implemented with minor modifications to the existing standard specification. An advantage of the pyramidal approach is that WSIs are treated equal to other DICOM imagery residing within the same server. In the JPIP-based approach, an additional JPIP server must be set up within the PACS. However, a disadvantage of the pyramidal approach is that because WSIs are stored in a DICOM-specific data structure using lossy compression, as is required in virtual microscopy, relocating WSIs from the PACS (e.g., for teaching purposes) requires lossy recompression. This results in image quality degradation. In the JPIP-based approach, on the other hand, the JPEG2000 WSIs are not stored using a DICOM-specific data structure, making them directly interchangeable with non-DICOM systems. Moreover, since patient-related DICOM data is not embedded in the JPEG2000 WSI files, the same JPIP server can be readily used inside and outside of a PACS without the need for anonymization.

Since the DICOM standard specification already includes support for JPIP, the pyramidal approach and the JPIP-based approach for whole-slide imaging are not mutually exclusive and can be used simultaneously in the same PACS. Regardless of the approach, changes in existing PACS workstation software are also required because of the WSI-specific image viewing characteristics. At the time of preparation of this article, the detailed contents of the upcoming WG-26 WSI supplement, as well as its expected release date, are open. In the future, when the supplement is finalized and implemented in practice, comparisons between the two WSI approaches might turn out useful.

\section{CONCLUSIONS}

To our knowledge, the software package described in this study is the first practical solution to overcome the limitations of DICOM in virtual microscopy. Compared to other approaches, such as the pyramidal approach of the DICOM WG-26, JPEG2000 with JPIP is a good alternative, enabling use of WSI archives either with or without a linked DICOM system. Further, since JPEG2000 has also many other advantageous features over those of existing WSI image formats, we anticipate that JPEG2000 will become a widely-accepted standard WSI format in virtual microscopy.

\section{ACKNOWLEDGMENTS}

This study was financially supported by the Finnish Cancer Foundation, Sigrid Juselius Foundation, and Tampere University Hospital with a governmental grant (EVO). The authors wish to thank Anna-Leena Lääperi from Tampere University Hospital and COST Action IC604: Eurotelepath ("Telepathology Network in Europe") for supportive collaboration.

\section{REFERENCES}

1. Digital Imaging and Communications in Medicine (DICOM). Available at ftp://medical.nema.org/dicom/2008/. Accessed 9 October 2008

2. Rojo MG, García GB, Mateos CP, García JG, Vicente MC: Critical comparison of 31 commercially available digital slide systems in pathology. Int J Surg Pathol 14(4):285-305, 2006

3. Weinstein RS: Innovations in medical imaging and virtual microscopy. Hum Pathol 36(4):317-9, 2005

4. Gilbertson J, Yagi Y: Histology, imaging and new diagnostic work-flows in pathology. Diagn Pathol 3(Suppl 1):S14, 2008

5. Lundin M, Lundin J, Helin H, Isola J: A digital atlas of breast histopathology: an application of web based virtual microscopy. J Clin Pathol 57(12):1288-1291, 2004

6. Lundin M, Lundin J, Isola J: Virtual microscopy. J Clin Pathol 57(12):1250-1, 2004

7. Ho J, Parwani AV, Jukic DM, Yagi Y, Anthony L, Gilbertson JR: Use of whole slide imaging in surgical pathology quality assurance: design and pilot validation studies. Hum Pathol 37(3):322-31, 2006

8. Kalinski T, Zwönitzer R, Sel S, Evert M, Guenther T, Hofmann H, Bernarding J, Roessner A: Virtual 3D microscopy using multiplane whole slide images in diagnostic pathology. Am J Clin Pathol 130(2):259-64, 2008

9. Gao M, Bridgman P, Kumar S: Computer aided prostate cancer diagnosis using image enhancement and JPEG2000. Proc SPIE 5203:323-334, 2003

10. Tuominen VJ, Isola J: The application of JPEG2000 in virtual microscopy. J Digit Imaging, doi:10.1007/s10278-0079090-z, 2007 
11. Taubman DS, Marcellin MW: JPEG2000: Image Compression Fundamentals, Standards and Practice, Boston: Kluwer Academic, 2002

12. The Joint Photographic Experts Group Web site. Available at http://www.jpeg.org/. Accessed 19 October 2008

13. Information technology_JPEG 2000 image coding system: Core coding system. ISO/IEC 15444-1, 2000 | ITU-T Recommendation T.800, 2002

14. Information technology_JPEG 2000 image coding system: Extensions. ISO/IEC 15444-2, 2002 | ITU-T Recommendation T.801, 2002

15. Information technology_JPEG 2000 image coding system: Interactivity tools, APIs and protocols. ISO/IEC 15444-9, 2005 | ITU-T Recommendation T.808, 2005

16. Della Mea V, Bortolotti N, Beltrami CA: A survey on non specialized off-the-shelf JPEG2000 viewers for digital microscopy use. Diagn Pathol 3(Suppl 1):S20, 2008

17. Kayser K, Görtler J, Goldmann T, Vollmer E, Hufnagl P, Kayser G: Image standards in Tissue-Based Diagnosis (Diagnostic Surgical Pathology). Diagn Pathol 3:17, 2008

18. Digital Imaging and Communications in Medicine (DICOM), Part 3: Information Object Definitions. Available at ftp://medical.nema.org/medical/dicom/2008/08_03pu.pdf. Accessed 9 October 2008

19. Digital Imaging and Communications in Medicine (DICOM), Correction Item CP-896: "Eliminate 16 bit row and column image size restrictions" (Letter Ballot). Available at ftp:// medical.nema.org/medical/dicom/CP/cp896_lb.pdf. Accessed 23 February 2009

20. Digital Imaging and Communications in Medicine (DICOM), Working Group 6 (Base Standard) meeting minutes, January 19-23, 2009, San Francisco, CA, USA. Available at http:// medical.nema.org/Dicom/minutes/WG-06/2009/2009-01-19/WG06_2009-01-19_Min.doc. Accessed 23 February 2009

21. Digital Imaging and Communications in Medicine (DICOM), Supplement 61: JPEG 2000 Transfer Syntaxes. Available at ftp://medical.nema.org/medical/dicom/final/sup61 ft. pdf. Accessed 9 October

22. Digital Imaging and Communications in Medicine (DICOM), Supplement 105: JPEG 2000 Part 2 Multi-component Transfer Syntaxes. Available at ftp://medical.nema.org/ medical/dicom/final/sup105 ft.pdf. Accessed 9 October 2008

23. Digital Imaging and Communications in Medicine (DICOM), Supplement 106: JPEG 2000 Interactive Protocol. Available at ftp://medical.nema.org/medical/dicom/final/ sup106_ft.pdf. Accessed 9 October 2008
24. The Internet Engineering Task Force (IETF), RFC 1951, DEFLATE Compressed Data Format Specification version 1.3. Available at http://www.ietf.org/rfc/rfc1951.txt. Accessed 9 October 2008

25. The Internet Engineering Task Force (IETF), RFC 2616, Hypertext Transfer Protocol - HTTP/1.1. Available at http:// www.ietf.org/rfc/rfc2616.txt. Accessed 9 October 2008

26. Taubman DS, Prandolini R: Architecture, philosophy and performance of JPIP: Internet protocol standard for JPEG2000. Proc SPIE 5150:791-805, 2003

27. Tian Y, Cai W, Sun J, Zhang J: A novel strategy to access high resolution DICOM medical images based on JPEG2000 interactive protocol. Proc SPIE 6919:691912, 2008

28. Peces C, García-Rojo M, Sacristán J, Gallardo AJ, Rodríguez A: Serendipia: Castilla-La Mancha telepathology network. Diagn Pathol 15(Suppl 1):S5, 2008

29. Kuratorium OFFIS e.V., Germany: DCMTK DICOM toolkit. Available at http://dicom.offis.de/. Accessed 9 October 2008

30. Qt Software, Norway: Qt cross-platform software development framework. Available at http://trolltech.com/products/. Accessed 9 October 2008

31. Tango Desktop Project, Tango Icon Library. Available at http://tango.freedesktop.org/Tango_Icon_Library. Accessed 9 October 2008

32. Aware Systems, Belgium: The BigTIFF File Format Proposal. Available at http://www.awaresystems.be/imaging/ tiff/bigtiff.html. Accessed 9 October 2008

33. Rasband WS, U.S. National Institutes of Health, USA: ImageJ image analysis software. Available at http://rsb.info.nih gov/ij/. Accessed 9 October 2008

34. Digital Imaging and Communications in Medicine (DICOM), Strategic Document \& Principal Contacts. Available at http://medical.nema.org/dicom/geninfo/Strategy.pdf. Accessed 9 October 2008

35. Digital Imaging and Communications in Medicine (DICOM), Supplement 122: Specimen Module and Revised Pathology SOP Classes. Available at ftp://medical.nema.org/ medical/dicom/final/sup122_ft2.pdf. Accessed 9 October 2008

36. Zwönitzer R, Kalinski T, Hofmann H, Roessner A, Bernarding J: Digital pathology: DICOM-conform draft, testbed, and first results. Comput Methods Programs Biomed, 87(3):181-8, 2007

37. Digital Imaging and Communications in Medicine (DICOM), Working Group 26 (Pathology) meeting minutes, May 17, 2008, Toledo, Spain. Available at http://medical.nema. org/DICOM/minutes/WG-26/2008/2008-05-17/WG-26_200805-17_Min.doc. Accessed 23 February 2009 